\title{
MORBILIDAD Y CICLOS TEMPORALES EN LA CONSULTA EXTERNA DE LA UNIVERSIDAD ESTATAL A DISTANCIA DE COSTA RICA
}

\author{
MS.O. Lourdes Arce Espinoza ${ }^{1}$, M.Sc. Julián Monge-Nájera ${ }^{2}$ \\ ${ }^{1}$ Enfermera, Servicios Médicos, UNED, larce@uned.ac.cr \\ 2 Investigador, Vicerrectoría Investigación,UNED, julianmonge@gmail.com \\ San José, Costa Rica \\ 2007
}

RESUMEN

Aparentemente, no existen datos publicados sobre la morbilidad y consulta externa del personal en una universidad a distancia. Este informe presenta esos datos para la Universidad Estatal a Distancia de Costa Rica. Los participantes en el estudio fueron todos los empleados que se presentaron a consulta entre el 15 de enero del 2004 y el 15 de diciembre del 2006. En lugar de hacer un muestreo, se estudió el total de la población, usando como instrumentos los 1526 expedientes médicos correspondientes. Procedimiento: en una primera etapa se leyeron los expedientes en su totalidad y se tabularon los datos de morbilidad. A partir de este registro se procedió al análisis estadístico con el programa Statgraphics Centurion XV. Los hallazgos principales fueron que el $50 \%$ de los pacientes solamente asistieron una vez a consulta externa durante el periodo y que la mayoría tenía entre 20 y 50 años de edad. Los días con mayor cantidad de consultas fueron lunes, martes y miércoles. La cantidad de consultas es relativamente estable a lo largo del año, con un leve aumento en junio. Las tres principales causas de consulta fueron enfermedades de las vías respiratorias superiores, trastornos musculoesqueléticos y problemas de sistema digestivo irritable. Conclusiones principales: la mayoría de las consultas corresponden a los departamentos con mayor cantidad de personal, pero algunos departamentos tuvieron valores extremadamente altos o bajos de consultas per capita, tal vez debido a factores como patologías que necesitan control periódico, cercanía geográfica y problemáticas psicosociales de pacientes que crean un círculo vicioso por somatización de estos problemas. Se rechazó la hipótesis de que al ser esta una población de alto nivel educativo, diferiría en su morbilidad del promedio nacional, pero se mantuvo la hipótesis de que presenta ciclos semanales y anuales.

\section{MORBIDITY AND TEMPORARY CYCLES FOR THE OUTPATIENT SERVICE AT UNIVERSIDAD ESTATAL A DISTANCIA IN COSTA RICA}

\section{ABSTRACT}

\begin{abstract}
No data has apparently been published on morbidity and outpatient service of faculty and staff of a distance university. This report presents such data from the Universidad Estatal a Distancia (Costa Rican State University for Distance Education). The participants in this study were the employees who were outpatients between January 15, 2004 and December 15, 2006. Instead of using a sample, the entire population was studied with a total of 1,526 medical records. Procedure: During the first stage all of the medical records were read and the morbidity data was tabulated. Subsequently, this information was statistically analyzed using Statgraphics Centurion XV. The main findings were that $50 \%$ of the patients used the outpatient service only once during the study period and that most of them were between 20 and 50 years of age. The days with the most consultations were Mondays, Tuesdays, and Wednesdays. The number of consultations was relatively stable throughout the year, with a slight increase in June. The three main causes for visiting the service were: upper respiratory tract diseases, muscularskeletal disorders, and irritated digestive system problems. Main conclusions: Most of the consultations were from the departments with the more faculty and staff members; however, some departments had extremely high or low consultation rates per capita, maybe due to factors such as pathologies that require periodic control, geographical proximity, and psycho-social issues of patients who create a vicious circle due to the somatization of such problems. The hypothesis that this population's morbidity rate would differ from the national average because of its high educational level was rejected. Nevertheless, the hypothesis that there are weekly and yearly cycles was maintained.
\end{abstract}




\section{MHSalud}

URL www.una.ac.cr/mhsalud

\section{INTRODUCCIÓN}

La frecuencia con que se produce una enfermedad o anomalía de la salud, en una determinada población o área, se conoce como morbilidad. Gracias a su sistema de seguridad social, Costa Rica cuenta con detalladas estadísticas sobre la atención y morbilidad de pacientes de consulta externa (Moya, 2003). En general, existen importantes diferencias en los patrones según el nivel económico y educativo (Morgan, 1988). Por ejemplo, las personas de mayor nivel educacional ocupan más consultas por problemas del aparato circulatorio; en cambio, las de menor nivel educacional tienen una mayor morbilidad por accidentes y violencia (Moya, 2003). Las universidades son, por su naturaleza, instituciones que tienden a concentrar personal de alto nivel educacional, y por tanto, se espera que difieran del promedio nacional en cuanto a sus patrones de consulta externa y morbilidad.

Tradicionalmente, el estudiantado universitario ha sido sujeto de gran cantidad de estudios biomédicos debido a que es una población muy asequible para el personal de investigación (e.g. Chen y Tsai, 2007; Bonner et al., 2007). Sin embargo, si en lugar del estudiantado se desea conocer las características del personal universitario, la situación cambia: la búsqueda detallada en las bases de datos no produjo resultados.

La Universidad Estatal a Distancia tiene el segundo lugar en mayor cantidad de estudiantes a nivel nacional (unos 22 000) y cuenta con personal académico de nivel medio y alto, así como personal de apoyo administrativo de todos los niveles. Se evalúan dos hipótesis:

Por su importante proporción de personal con alto nivel educacional, la UNED difiere del patrón nacional, ya que se sabe que el nivel educacional afecta tanto las causas de morbilidad como la consulta externa (Moya, 2003)

En la UNED, la consulta externa presenta ciclos según el día de la semana y el mes, como se ha encontrado en la población general de diversos países (Seretakis et al., 1997; Magid et al., 2005).

No existen datos publicados que permitan evaluar estas hipótesis. Este trabajo presenta el primer estudio sobre las causas de consulta externa del personal de una universidad a distancia, según morbilidad, y sobre sus patrones según día de la semana y mes.

\section{METODOLOGÍA}

Materiales: La recolección de los datos se realizó de una fuente primaria de información: el expediente médico, de manera retrospectiva, pues las variables se codificaron y tabularon al final del periodo de estudio. Se usaron además la hoja electrónica Excel y Programa estadístico Statgraphics Centurion XV.

Sujetos: Los sujetos de estudio fueron todos los pacientes de la consulta externa del servicio médico de la Universidad Estatal a Distancia, que consultaron entre el 15 de enero del 2004 y el 15 de diciembre del 2006 (total 1526 expedientes). La población estudiada presenta el siguiente perfil sociodemográfico: entre 20 y 70 años de edad, residentes principalmente del Gran Área Metropolitana, con nivel socioeconómico variable. Preparación académica variable, ya que abarcó desde personal de apoyo, como conserjes, hasta académicos, pero con mucha mayor proporción de personal de nivel universitario debido a que es una institución de educación superior. Los académicos tienen en buena parte estudios superiores (licenciaturas, maestrías y doctorados). La gran mayoría personas son casadas y con edad laboral en la institución de 5 a 30 años. 


\section{MHSalud}

URL www.una.ac.cr/mhsalud

Procedimiento: Se usó una metodología descriptiva e inferencial; la metodología descriptiva para presentar las consultas según su distribución por día, mes y departamento. La estadística inferencial se usó para identificar los patrones de los datos como parte de la evaluación de las dos hipótesis presentadas en la introducción. La recolección de datos se llevó a cabo en dos etapas: la primera comprendió la revisión del expediente médico; la segunda fue la digitación de los datos en la hoja electrónica. A partir de este registro se procedió a la codificación de los datos y a su análisis estadístico. Se trabajaron las categorías agrupadas por edad, día, mes, patología y departamento laboral, usando la prueba chicuadrado. Al usarse la totalidad de los expedientes médicos, se obtuvo la morbilidad de toda la población para el periodo considerado de dos años.

\section{RESULTADOS}

El 50\% de los pacientes solamente asistieron una vez a consulta externa durante el periodo. Aproximadamente el $25 \%$ hizo entre 3 y 17 consultas, el resto una cantidad mayor, hasta un máximo de 52 consultas (Cuadro 1).

Cuadro 1. Número de consultas por persona en la consulta externa de la Universidad Estatal a Distancia, 2004-2006

\begin{tabular}{cc}
\hline Número de consultas & Número de pacientes con esa cantidad de consultas \\
\hline 1 a 10 & 1111 \\
11 a 20 & 191 \\
21 a 30 & 94 \\
31 a 40 & 43 \\
más de 41 & 33 \\
\hline
\end{tabular}

La mayoría de los funcionarios tenía entre 20 y 50 años de edad al momento de la consulta, aunque hubo extremos cercanos a los 20 y a los 70 años $\left(X^{2}=807\right.$, grados de libertad 5, $\mathrm{p}<0.01$; Cuadro 2).

Cuadro 2. Edad de pacientes en la consulta externa de la Universidad Estatal a Distancia, 2004-2006

\begin{tabular}{cc}
\hline Edad & Número de personas \\
\hline 18 a 27 & 253 \\
28 a 37 & 341 \\
38 a 47 & 422 \\
48 a 57 & 355 \\
más de 58 & 64 \\
\hline
\end{tabular}

Los días con mayor cantidad de consultas fueron lunes, martes y miércoles. La cantidad de pacientes disminuyó al acercarse el fin de semana $\left(X^{2}=235\right.$, grados de libertad $4, p<0.01$; Gráfico 1). 


\section{MHSalud}

URL www.una.ac.cr/mhsalud

Gráfico 1. Días de más consultas en la consulta externa de la Universidad Estatal a Distancia, 2004-2006 (cantidad acumulada de consultas)

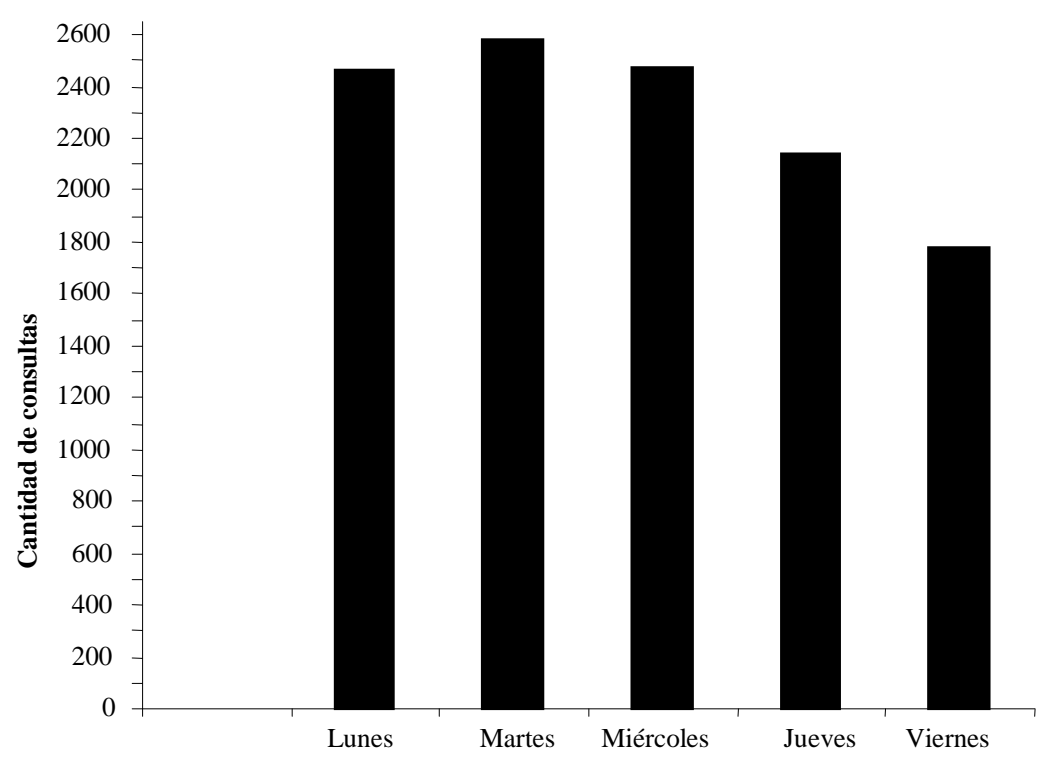

Días de la semana

Como la UNED cierra dos semanas en diciembre, una en enero y en abril, puede considerarse que la cantidad de consultas es relativamente estable a lo largo del año, pero con un aumento en junio $\left(\mathrm{X}^{2}=182\right.$, grados de libertad 9, $\mathrm{p}<0.01$; Gráfico 2$)$.

Gráfico 2. Distribución mensual de las consultas en la consulta externa de la Universidad Estatal a

Distancia, 2004-2006.

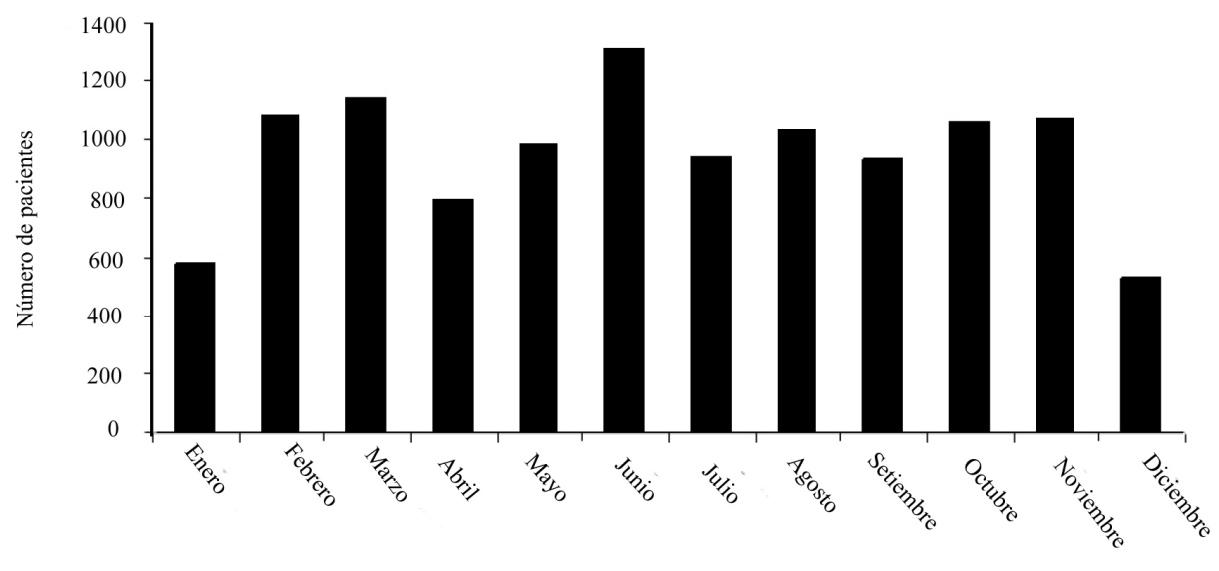

Meses 


\section{MHSalud}

URL www.una.ac.cr/mhsalud

Las 10 principales causas de consulta son: enfermedades de las vías respiratorias superiores, trastornos musculoesqueléticos, problemas de sistema digestivo irritable, hipertensión arterial, dislipidemias, trauma, dermatitis y enfermedad diarreica aguda. También hay una cantidad importante de consultas que se hacen como requisito del certificado médico de ingreso (personal nuevo) y de consultas en las cuales la persona se diagnostica como "adulto sano" $\left(X^{2}=2794360\right.$, grados de libertad 74, $p<0.01$; Cuadro 3).

Cuadro 3. Distribución de los diagnósticos según su frecuencia en la consulta externa de la Universidad Estatal a Distancia, 2004-2006

\begin{tabular}{|c|c|}
\hline Diagnóstico & Número de pacientes \\
\hline Absceso & 61 \\
\hline Accidente laboral & 3 \\
\hline Acné & 13 \\
\hline Adenopatía & 24 \\
\hline Adinamia & 42 \\
\hline Adulto sano & 436 \\
\hline Alopecia & 5 \\
\hline Amenaza de aborto & 12 \\
\hline Asma & 66 \\
\hline Cáncer & 30 \\
\hline Cefalea & 251 \\
\hline Celulitis & 98 \\
\hline Certificado lactancia & 13 \\
\hline Cirugía menor & 115 \\
\hline Citología vaginal & 200 \\
\hline Control pos-operatorio & 7 \\
\hline Control prenatal & 142 \\
\hline Crisis convulsiva & 3 \\
\hline Cuerpo extraño & 19 \\
\hline Dengue & 3 \\
\hline Depresión & 149 \\
\hline Dermatitis & 382 \\
\hline Diabetes mellitus & 205 \\
\hline Digestivo irritable & 819 \\
\hline Discapacidad física permanente & 3 \\
\hline Dislipidemia & 451 \\
\hline Drogodependencia & 6 \\
\hline Enfermedad diarreica aguda & 293 \\
\hline Enfermedades de encías y boca & 64 \\
\hline Enfermedades de transmisión sexual & 5 \\
\hline Estrés & 6 \\
\hline Examen de ingreso & 663 \\
\hline Fiebre origen desconocido & 8 \\
\hline Hemorroides & 62 \\
\hline Herpes & 38 \\
\hline Hipertensión arterial & 710 \\
\hline
\end{tabular}




\section{MHSalud}

URL www.una.ac.cr/mhsalud

Cuadro 3. Continuación

Infección de las vías respiratorias inferiores

Infección de las vías respiratorias superiores

Lipoma

Lipotimia

Menopausia

Micosis

Obesidad

100

Onicocriptosis

Otros

Parasitosis externa

Pitiriasis versicolor

Planificación

Psoriasis

Rinitis

Síndrome vertiginoso

Tapón cerumen

Tos

Trastorno bipolar

Trastorno obsesivo compulsivo

Trastornos ácido úrico

Trastornos auditivos

Trastornos cardiovasculares

Trastornos de la glándula tiroides

Trastornos del sueño

$\begin{array}{lr}\text { Trastornos genitourinarios femeninos } & 234\end{array}$

Trastornos genitourinarios masculinos 156

Trastornos ginecológicos $\quad 430$

$\begin{array}{lr}\text { Trastornos hematológicos } & 73\end{array}$

$\begin{array}{lr}\text { Trastornos hepáticos } & 37\end{array}$

Trastornos musculoesqueléticos 1171

$\begin{array}{ll}\text { Trastornos oftalmológicos } & 251\end{array}$

$\begin{array}{lr}\text { Trastornos reumatológicos } & 166\end{array}$

$\begin{array}{ll}\text { Trauma } & 412\end{array}$

Virosis $\quad 51$

La mayoría de las consultas corresponden a los departamentos de Servicios Generales, Editorial y diversas escuelas $\left(X^{2}=12631\right.$, grados de libertad 48, $\mathrm{p}<0.01$; Cuadro 4). 


\section{MHSalud}

URL www.una.ac.cr/mhsalud

Cuadro 4. Consultas por departamento en la consulta externa de la Universidad Estatal a Distancia, 2004-2006

\begin{tabular}{|c|c|}
\hline Departamento & $\begin{array}{c}\text { Total de consultas por } \\
\text { departamento }\end{array}$ \\
\hline Almacén General & 50 \\
\hline Archivo & 8 \\
\hline Asociación Solidarista de Empleados & 120 \\
\hline Asuntos Estudiantiles & 19 \\
\hline Audiovisuales & 205 \\
\hline Auditoría Interna & 43 \\
\hline Bienestar Estudiantil & 253 \\
\hline Centro de Capacitación en Educación a Distancia & 34 \\
\hline Centro de Información de Recursos Bibliográficos & 409 \\
\hline Centro de Investigación y Evaluación Institucional & 91 \\
\hline Centro de Operaciones Académicas & 278 \\
\hline Centro de Mejoramiento de la Producción Académica & 153 \\
\hline Centro Educación Ambiental & 25 \\
\hline Colegio de Educación a Distancia & 44 \\
\hline Consejo Nacional de Rectoría & 4 \\
\hline Consejo Universitario & 131 \\
\hline Contabilidad & 285 \\
\hline Contratación y Suministros & 205 \\
\hline Defensoría de los Estudiantes & 45 \\
\hline Dirección de Centros Universitarios & 443 \\
\hline Dirección de Tecnología e Información & 352 \\
\hline Dirección Financiera & 483 \\
\hline Dirección y Producción de Material Didáctico & 519 \\
\hline Distribución y Ventas & 288 \\
\hline Editorial & 705 \\
\hline Escuela Ciencias de Administración & 457 \\
\hline Escuela Ciencias de Educación & 560 \\
\hline Escuela Ciencias Exactas y Naturales & 614 \\
\hline Escuela Ciencias Sociales y Humanidades & 620 \\
\hline Librería & 41 \\
\hline Mercadeo y Comunicación & 141 \\
\hline Oficina Jurídica & 62 \\
\hline Pensionado & 56 \\
\hline Presupuesto & 280 \\
\hline Producción Académica & 48 \\
\hline Rectoría & 44 \\
\hline Recursos Humanos & 530 \\
\hline Registro & 485 \\
\hline Salud Ocupacional & 27 \\
\hline Servicio Médico & 5 \\
\hline Servicios Generales & 1247 \\
\hline Sindicato & 6 \\
\hline Sistema de Estudios de Posgrado & 305 \\
\hline
\end{tabular}

(cuadro continúa)

Revista MHSalud® (ISSN: 1659-097X) Vol. 5. No. 1. Julio, 2008. 


\section{MHSalud}

URL www.una.ac.cr/mhsalud

Cuadro 4. Continuación

\begin{tabular}{lc}
\hline Tesorería & 208 \\
Vicerrectoría Académica & 197 \\
Vicerrectoría de Planificación & 102 \\
Vicerrectoría Ejecutiva & 126 \\
Videoconferencias & 43 \\
\hline
\end{tabular}

Servicios Generales, la Editorial y las cuatro escuelas de la UNED son los departamentos con mayor cantidad de personal y tienen la mayor cantidad de consultas (Cuadro 6). Si se divide esta cantidad entre la de empleados en cada uno, los tres departamentos con mayor cantidad de consultas por empleado son Registro, Mercadeo y Salud Ocupacional (Cuadro $5)$.

Cuadro 5. Consultas por persona según departamento de trabajo en la consulta externa de la Universidad Estatal a Distancia, 2004-2006

\begin{tabular}{|c|c|}
\hline Departamento & Consultas/empleado \\
\hline Asociación Solidarista Empleados & 10,00 \\
\hline Asuntos Estudiantiles & 1,12 \\
\hline Auditoría Interna & 8,60 \\
\hline Bienestar Estudiantil & 9,37 \\
\hline Centro de Capacitación en Educación a Distancia & 4,25 \\
\hline Centro de Información de Recursos Bibliográficos & 24,53 \\
\hline Centro de Investigación y Evaluación Institucional & 8,27 \\
\hline Centro de Mejoramiento de la Producción Académica & 12,75 \\
\hline Centro de Operaciones Académicas & 21,38 \\
\hline Centro Educación Ambiental & 4,17 \\
\hline Colegio de Educación a Distancia & 1,91 \\
\hline Consejo Universitario & 18,71 \\
\hline Contabilidad & 19,00 \\
\hline Contratación y Suministros & 20,50 \\
\hline Defensoría de los Estudiantes & 22,50 \\
\hline Dirección de Centros Universitarios & 2,31 \\
\hline Dirección de Extensión & 2,86 \\
\hline Dirección de Tecnología e Información & 9,26 \\
\hline Dirección Financiera & 12,00 \\
\hline Dirección y Producción de Material Didáctico & 16,67 \\
\hline Distribución y Ventas & 13,16 \\
\hline Editorial & 13,56 \\
\hline Escuela Ciencias de Administración & 3,91 \\
\hline Escuela Ciencias de Educación & 3,61 \\
\hline Escuela Ciencias Exactas y Naturales & 3,01 \\
\hline Escuela Ciencias Sociales y Humanidades & 3,32 \\
\hline Mercadeo y Comunicación & 28,20 \\
\hline Oficina Jurídica & 15,50 \\
\hline Presupuesto & 16,47 \\
\hline
\end{tabular}




\section{MHSalud}

URL www.una.ac.cr/mhsalud

\begin{tabular}{lc} 
Cuadro 5. Continuación & \\
\hline Producción Académica & 4,00 \\
Rectoría & 4,40 \\
Recursos Humanos & 14,32 \\
Registro & 34,64 \\
Salud Ocupacional & 27,00 \\
Servicios Generales & 13,80 \\
Sistema de Estudios de Postgrado & 2,68 \\
Tesorería & 17,33 \\
Vicerrectoría Académica & 1,74 \\
Vicerrectoría de Planificación & 12,75 \\
Vicerrectoría Ejecutiva & 14,56 \\
\hline
\end{tabular}

\section{DISCUSIÓN}

Número de consultas por persona: La mitad de las consultas corresponde a personas que llegaron al servicio médico una sola vez durante el periodo. Para entender porqué lo hicieron solo una vez, se revisó los expedientes y se encontró que en general se trata del personal nuevo, que debe hacer una consulta obligatoria para obtener el "certificado médico de ingreso". Se cree que mucho de este personal nuevo trabaja también en otras instituciones y suele hacer su consulta externa en otras entidades de salud.

El 25\% de la población, que hizo 2-18 consultas en los dos años, corresponde en general a controles de salud de patologías diversas, pero en todo caso predomina la policonsulta: "el paciente policonsultante se define como aquella persona que recurre con una frecuencia de siete o más consultas al año y cuya asistencia es por iniciativa propia" (Caja Costarricense de Seguro Social, 2002).

Edad: Como el personal ingresa alrededor de los 20 años de edad y se pensiona (durante el periodo estudiado) poco después de los 50 años, casi un 50\% de los pacientes se encuentra dentro de ese ámbito de edades, que corresponde al económicamente más activo y también a la mayoría de las consultas externas a nivel nacional (Moya, 2003). Los dos picos de edad en la consulta corresponden a la de ingreso como empleados nuevos (examen obligatorio) y a la del periodo de envejecimiento posterior a los 50 años de edad.

Días de más consultas (2004-2006): La mayor frecuencia de consulta de lunes a miércoles coincide con la tendencia nacional, ya que en Costa Rica hay más consulta al iniciarse la semana (Moya, 2003). Se propone como hipótesis para futuros estudios la siguiente explicación. Como el consultorio no abre los fines de semana, se produce un efecto acumulativo: si algo les ocurre el fin de semana, los funcionarios esperan hasta el lunes. Pero el día con más citas es el martes, esto tal vez porque los pacientes dedican el lunes a ponerse al día con sus tareas, por lo cual se sobrecarga el martes. Los jueves y viernes, cuando se acerca el fin de semana, baja el número de consultas, tal vez porque esos días son frecuentes los permisos y vacaciones (observación personal de la primera autora). En otros países se ha notado que la mortalidad aumenta en los días feriados y lunes, por el menor acceso a servicios de salud y por la tensión sicológica del regreso al trabajo (Magid et al., 2005). En la UNED la tasa de mortalidad es tan baja que no es posible identificar un patrón semejante. 


\section{MHSalud}

URL www.una.ac.cr/mhsalud

Meses con más consultas (2004-2006): Considerando el cierre por Navidad y Semana Santa, el único efecto estacional notable es un aumento de la consulta en junio. Se propone como hipótesis la activación de las enfermedades de las vías respiratorias y diarreicas típicas en esta época del año. Es bien conocida la estacionalidad en este tipo de enfermedades, así como en las del sistema cardiovascular (Seretakis et al., 1997).

Diagnóstico de causas de morbilidad: La predominancia de consultas por enfermedades de las vías respiratorias superiores, trastornos musculoesqueléticos, problemas de sistema digestivo irritable, hipertensión arterial, dislipidemias, trauma, dermatitis y enfermedad diarreica aguda, coincide con las causas más comunes de consulta a nivel nacional publicadas por Moya (2003). Estos patrones se asocian normalmente con factores como edad y actividad económica (Bravo y Vargas, 1991). La gran cantidad de pacientes que son diagnosticados como "adulto sano" no representan un problema de consultas injustificadas. Ellos lo hacen por control de salud, específicamente solicitud o revisión de resultados de laboratorio que se hallan dentro de los parámetros normales. Los exámenes médicos son un requisito administrativo de ingreso para los nuevos empleados de la UNED, gran cantidad de ellos son profesores o tutores, por lo que estos también producen muchas consultas a pesar de estar sanos.

Consultas totales por departamento: La mayoría de las consultas corresponden a los departamentos con mayor cantidad de personal, que son Servicios Generales, Editorial y las cuatro escuelas de la UNED. Como la consulta externa de esta universidad se acerca a un nivel peligroso de saturación, se sugiere que se busquen mecanismos para disminuir la demanda por parte de estos seis departamentos.

Consultas por persona según departamento: Comparando estudiantes que viven fuera de la universidad con los que viven en ella, se ha demostrado que la convivencia en las instalaciones favorece el contagio de enfermedades (Magid et al., 2005). En este caso, no se estudia las causas de que algunos departamentos tuvieran valores extremadamente altos o bajos de consultas per capita, pero posibles factores que se recomienda estudiar son: seguimiento de patologías crónicas u otras aquellas que necesiten control periódico, cercanía geográfica, hacinamiento, estrés y carga laboral, búsqueda de apoyo para solución de problemas personales laborales, familiares o sociales y finalmente excesiva atención a las problemáticas psicosociales de pacientes que crean un círculo vicioso, cuando se consulta por somatización.

\section{CONCLUSIONES}

La mitad de los pacientes solamente asistieron una vez a consulta externa durante el periodo estudiado.

La mayoría de los pacientes tenía entre 20 y 50 años de edad al momento de la consulta.

Los días con mayor cantidad de consultas fueron lunes, martes y miércoles.

La cantidad de consultas es relativamente estable a lo largo del año, con un ligero aumento en junio.

Las tres principales causas de consulta fueron enfermedades de las vías respiratorias superiores, trastornos músculo-esqueléticos y problemas de sistema digestivo irritable. 


\section{MHSalud}

URL www.una.ac.cr/mhsalud

La mayoría de las consultas corresponden a los departamentos con mayor cantidad de personal.

Algunos departamentos tuvieron valores extremadamente altos o bajos de consultas per capita.

Se rechaza la hipótesis de que la consulta externa y su morbilidad en la UNED siguen un patrón diferente del nacional, debido a que tiene una mayor proporción de personas con alto nivel educacional.

Se mantiene la hipótesis de que la consulta externa y su morbilidad en la UNED presentan ciclos semanales y anuales.

\section{REFERENCIAS}

Bonner, A., Macculloch, P., Gardner, T., y Chase, C.W. (2007). A student-led demonstration project on fall prevention in a long-term care facility. Geriatric Nursing, 28, 312318.

Bravo, J., y Vargas, N. (1991). Tendencias y fluctuaciones de la morbilidad y la mortalidad por ciertas causas y la actividad económica; Costa Rica, Chile y Guatemala, 19601986. Notas de Población Chile, 19, 117-146.

Caja Costarricense de Seguro Social. (2002). Modernización: Comunicación para un mejor cambio. Boletín Caja Costarricense de Seguro Social. San José, Costa Rica.

Chen, R.S. y Tsai, C.C. (2007). Gender differences in Taiwan university students attitudes toward web-based learning. Cyberpsychologic and Behavior, 10, 645-654.

Magid, J. D., Yongfei, W., Herrin, J., McNamara, L. R., Bradley, H. E., Curtis, P.J., Pollack, V. C., French, J. W., Blaney, E. M., y Krumholz, M. H. (2005). Relationship between time of day, day of week, timeliness of reperfusion, and inhospital mortality for patients with acute ST-Segment elevation myocardial infarction. Journal of the American Medical Association, 294, 803-812.

Morgan, L. (1988). Salud sin riqueza: el sistema de salud en Costa Rica bajo la crisis económica. Revista Centroamericana de Administración Pública, 12, 25-27.

Moya, L. (2003). Cambios en la morbilidad y mortalidad por edad y sexo, Costa Rica, 1987, 1992, 1997 y 2002. Sección de Información Biomédica, Caja Costarricense de Seguro Social, San José, Costa Rica.

Seretakis, D., Lagiou, P., Lipworth, L., Signorello, L. B., Rothman, K. J., y Trichopoulos, D. (1997). Changing seasonality of mortality from coronary heart disease. Journal of the American Medical Association, 278, 1012-1014. 


\section{MHSalud}

URL www una.ac.cr/mhsalud

Fecha de recepción del artículo: 01 de noviembre del 2007.

Fecha de aceptación del artículo: 01 de febrero del 2008.

Fecha de publicación del artículo: 31 de julio del 2008. 